\title{
Autonomic function tests: introduction to the series
}

\author{
David S. Goldstein ${ }^{1}$ William P. Cheshire Jr. ${ }^{2}$
}

Received: 7 March 2017/ Accepted: 8 March 2017/Published online: 17 March 2017

(C) Springer-Verlag Berlin Heidelberg (outside the USA) 2017

\section{Introduction to the series on autonomic function testing}

Clinical Autonomic Research is initiating a series on autonomic function testing. In this introduction to the series, we lay out the rationale for the series, why it is timely, the approach, the issue of research vs. medical practice, types of autonomic function testing modalities, and a planned listing of topics to be covered.

\section{Rationale for the series}

The discipline of autonomic medicine utilizes a wide range of tests for research and clinical care, and different centers offer different tests. These include tests of anatomical structures of autonomic nerves, ganglia, and central nervous system regions, as well as functional tests of sympathetic, parasympathetic, and enteric autonomic responses. As patients with autonomic disorders often present with a constellation of autonomic symptoms, and no one test evaluates the autonomic nervous system in its entirety, most centers offer batteries of autonomic tests. The selection of tests differs from center to center depending on the specialties and clinical interests of

David S. Goldstein

goldsteind@ninds.nih.gov

1 Clinical Neurocardiology Section, Clinical Neurosciences Program, Division of Intramural Research, National Institute of Neurological Disorders and Stroke, National Institutes of Health, 9000 Rockville Pike MSC-1620, Building 10 Room 5N220, Bethesda, MD 20892, USA

2 Department of Neurology, Mayo Clinic, Jacksonville, FL 32224, USA autonomic investigators and the types of clinical disorders and symptomatic concerns that come to their attention. These tests differ in methodology, validity, sensitivity, and interpretation.

Clinicians need to know which tests yield what kinds of information about which autonomic disorders if they are to reach accurate diagnoses, develop sound treatment plans, and educate their patients. Students, residents, and fellows need guidance in interpreting autonomic test results so that they can learn to recognize autonomic disorders, recommend physiologically-based therapies, and obtain appropriate consultations. Third party payers also need published evidence and expert guidelines that address risk vs. benefit and expense vs. value to deal fairly and economically with claims for reimbursement of services.

Some tests are by now outdated or have been superseded, some are more informative than others, and some are relatively new and promising but not widely available.

\section{Approach}

The approach will be didactic, the goal being to teach clinicians and trainees as well as academicians. In order to make the points "sticky," the series will rely heavily on anecdote, analogy, and art.

\section{Why is this series timely?}

Autonomic medicine is a relatively new discipline. Some of the conditions that come under the umbrella of "dysautonomia" have only recently been identified.

Dysautonomias are a difficult topic, for several reasons. They are multidisciplinary, integrative, mind-body 
disorders. Different centers have different emphases. Disorders of the autonomic nervous system are not included in most medical school curricula.

Nevertheless, dysautonomias are common, often debilitating, and an increasing public health burden [1-3]. The clinical demand is huge. Websites, blogs, and other Internet sources of information (and misinformation) are mushrooming, and it is becoming increasingly difficult to separate fact from fiction. These recent developments make it increasingly important to understand the bases, strengths, and weaknesses of available tests. As will become clear, the field of autonomic function testing has also unfortunately been a platform for charlatanism, and it is important and timely to set the record straight.

With the relatively recent accreditation of fellowships in autonomic disorders by the United Council for Neurologic Subspecialties, it is also important for autonomic fellows to have a reliable resource about autonomic function tests in order for them to prepare for the certifying examination.

\section{What is research and what is medical practice?}

In the United States, medical billing is disproportionately test- and procedure-based. Coding for reimbursements by third party payers or insurance carriers is the complex product of many economic factors that can have little to do with clinical importance. One basis for rejecting reimbursement claims is that autonomic function tests are "research" or "investigational" and allegedly not part of established medical practice.

The United States Code of Federal Regulations defines "research as a systematic investigation designed to develop or to contribute to generalizable knowledge" [4]. Autonomic testing is useful both as a research tool and, in the case of established methodologies, also in patient care.

"Medicine," according to a leading textbook, "is a profession that incorporates science and the scientific method with the art of being a physician" [5]. Medicine thus encompasses both clinical practice and medical research. To the greatest degree possible, medical practice is evidence-based, employing the conscientious and judicious use of current best evidence, as guided by clinical experience and within the framework of the patient's values, to reach medical decisions. Whereas randomized controlled trials constitute the strongest evidence, this level of evidence does not always exist for autonomic disorders that are understudied, rare, or only recently described. In evaluating autonomic tests, the best available evidence may comprise prospective or descriptive small series, retrospective series, qualitiative research, or case reports. Until larger studies and outcome studies are available, best practices and the standard of care for patients with autonomic disorders rely on expert opinion based on clinical experience, knowledge of physiology, and scientific principles.

This series will reinforce the appropriateness of tests that are already covered but also present the case that other tests should be covered because of their value in diagnosis, tracking treatment, or predicting outcome.

\section{Topics to be covered}

The series begins with the medical history-by far the most valuable (and likely the most poorly reimbursed) autonomic function test. Taking a proper autonomic history requires intelligent and clinically astute probing, an inquisitive attitude, and time to sort through a symptom list that may at first seem vague, muddled, or elusive. This is not a problem to be solved by checklists or symptom inventories or something that a technician can perform. As will be explained in detail, the autonomic Review of Systems is not nearly as informative as the History of the Present Illness.

The next topic will be monitoring responses of beat-tobeat blood pressure and heart rate to the Valsalva maneuver. In the past this test required an indwelling intra-arterial catheter; however, with some exceptions, one can track beat-to-beat blood pressure accurately and non-invasively such as by using an automated finger cuff device.

Subsequent topics will be tilt table testing, heart rate variability, sweat tests, neuropharmacologic probes, clinical neurochemistry, autonomic neuroimaging, skin biopsies, and genetics.

\section{Overview of autonomic function tests}

Tests for dysautonomias can be divided into physiological, neuropharmacologic, neurochemical, neuroimaging, and genetic.

Physiological tests involve measurements of a body function in response to a manipulation such as the Valsalva maneuver, standing, tilt table-testing, or altered temperature at the skin.

Neuropharmacologic probes involve administering a drug and measuring its effects either on a physiological measure (e.g., blood pressure) or a biochemical measure (e.g., the plasma norepinephrine concentration). There always is at least some risk of autonomic function testing using neuropharmacologic probes, not only because of side effects of the test drugs but also because the drugs can interact with medications the patient is on 
to treat the disease or with other conditions the patient has.

Neurochemical tests involve measuring levels of body chemicals, such as the catecholamines, norepinephrine and adrenaline, either under resting conditions or in response to physiological or neuropharmacologic manipulations. Several factors influence plasma norepinephrine levels besides release from sympathetic nerves. Simultaneous measurements of deaminated metabolites of the catecholamines and of the catecholamine precursor DOPA can be more informative than measurements of the catecholamines in isolation.

Neuroimaging tests, which are relatively new, involve visualizing the autonomic nerve supply in body organs. Central nervous system neuroimaging can also be used to identify brain diseases that are associated with dysautonomias. For instance, different types of scans can identify the loss of nerve terminals that contain dopamine in the brain in Parkinson's disease or identify abnormalities of brain structures that regulate the autonomic nervous system.

Examining nerves in skin biopsy specimens by immunohistochemistry or immunofluorescence microscopy can be considered to be another sort of neuroimaging. This has the potential advantage of visualizing autonomic innervation simultaneously with other proteins-especially alpha synuclein, the defining protein in autonomic synucleinopathies.

Genetic tests involve analyses of genetic material for abnormalities of specific genes or gene expression that produce or predispose to the development of particular diseases. Rarely—but importantly—genotypic abnormalities are causally related to autonomic failure.

\section{Historical perspective}

Because of these new technologies, the area of autonomic function tests is a rapidly evolving field. In this series we will include historical perspective, so that readers gain insight into how we reached the current situation, what the issues and controversies are presently, and where we think the field is headed.

Acknowledgements The research reported here was supported by the Division of Intramural Research of the NINDS.

Financial support Division of Intramural Research, NINDS, NIH.

\section{Compliance with ethical standards}

Conflict of interest The corresponding author states that there is no conflict of interest.

\section{References}

1. Cheshire WP (2016) Autonomic disorders and their management. In: Goldman, Schafer AI (eds) Goldman-Cecil Medicine, 25th edn. Elsevier Saunders, Philadelphia, pp 2517-2522

2. Low PA, Benarroch EE (2008) Clinical autonomic disorders: evaluation and management, 3rd edn. Lippincott Williams \& Wilkins, Philadelphia

3. Iodice V, Sandroni P (2014) Autonomic neuropathies. Continuum 20:1373-1397

4. https://www.hhs.gov/ohrp/regulations-and-policy/regulations/45cfr-46/index.html\#46.102

5. Goldman L, Schafer AI (2016) Approach to medicine, the patient, and the medical profession. In: Goldman L, Schafer AI (eds) Goldman-Cecil Medicine, 25th edn. Elsevier Saunders, Philadelphia, p 1 